\title{
Las sentencias estructurales del Tribunal Constitucional peruano en el ámbito de los derechos socioeconómicos: salud y educación(*)
}

\author{
The structural judgments of the Peruvian Constitutional Court in the field \\ of socioeconomic rights: health and education
}

\author{
Silvia Haydee Sánchez Gómez ${ }^{(*)}$ \\ Tribunal Constitucional del Perú (Lima, Perú)
}

\begin{abstract}
Resumen: En las últimas décadas se ha producido un fenómeno que alcanza a la jurisdicción constitucional de países con grandes desigualdades sociales. Parte de dicho fenómeno consiste en el uso de una figura creada jurisprudencialmente denominada sentencias estructurales, pasando por el caso Brown vs. Board of Education en los Estados Unidos debido a temas de segregación racial; Grootboom vs. República de Sudáfrica, por el derecho a una vivienda digna; y el de la Unión del Pueblo para las Libertades Civiles vs. Unión India y otros, por el derecho a una alimentación básica, hasta llegar a la sentencia T-025 de la Corte Constitucional de Colombia sobre desplazamiento forzado de personas. En el Perú, ha sido el Tribunal Constitucional el que ha dictado, en más de una docena de oportunidades, este tipo de sentencias, las que han recaído básicamente sobre derechos socioeconómicos. Pese a ello, su desarrollo en nuestro medio aún es incipiente, por lo que resulta pertinente analizar el camino que viene transitando.
\end{abstract}

Palabras claves: Sentencias estructurales - Estado de cosas inconstitucionales Derechos sociales - Giro tutelar - Exigibilidad judicial - Derecho Constitucional.

\begin{abstract}
In recent decades there has been a phenomenon that reaches the constitutional jurisdiction of countries with large social inequalities. Part of this phenomenon is the use of a jurisprudentially created figure called a structural judgment. Since Brown vs. Board of Education in the United States due to issues of racial segregation; Grootboom vs. Republic of South Africa, for the right to decent housing and that of the People's Union for Civil Liberties vs. Indian Union and others, for the right to basic food, until reaching the judgment $\mathrm{T}-025$ of the Constitutional Court of Colombia on forced displacement of persons. In Peru, it has been the Constitutional Court that has issued more than a dozen occasions such judgments, which have fallen mainly on socio-economic rights. Despite this, its development in our environment is still incipient, so it is pertinent to analyze the path that is going.
\end{abstract}

Keywords: Structural remedies - State of unconstitutional affairs - Social rights Expansive effects of protection - Judicial enforceability - Constitutional Law.

(*) Nota del Editor: este artículo fue recibido el 23 de febrero de 2020 y su publicación fue aprobada el 4 de mayo de 2020.

${ }^{* *}$ ) Abogada por la UNMSM, máster y ex becaria en Derecho Constitucional por el Centro de Estudios Políticos y Constitucionales (CEPC) en convenio con la Universidad Internacional Menéndez Pelayo, máster en Derecho Público por la Universidad Carlos III de Madrid, egresada de la Maestría en Derecho Constitucional y Derechos Humanos por la UNMSM, asesora jurisdiccional del Tribunal Constitucional, ha sido profesora del curso de Derecho Procesal Constitucional de la UNMSM y actualmente es doctorando del Programa de Doctorado en Estudios Avanzados en Derechos Humanos de la Universidad Carlos III de Madrid. Contacto: sisa20_5@ hotmail.com 
Las sentencias estructurales del Tribunal Constitucional peruano en el ámbito de los derechos socioeconómicos: salud y educación

The structural judgments of the Peruvian Constitutional Court in the field of socioeconomic rights: health and education

\section{Introducción}

Hasta el año 2004, la Corte Constitucional de Colombia recibió decenas de procesos de tutela (amparos) que recayeron sobre todo en derechos económicos y sociales. Todos ellos tenían algo en común: su origen, esto es, la situación de desplazamiento forzado del que habían sido víctimas a causa de la violencia ejercida por las Fuerzas Armadas Revolucionarias de Colombia (FARC) y de los paramilitares. La Corte, en lugar de resolver estos casos de manera individual, optó por reunirlos todos en una macro sentencia y expedir un fallo en el que se declaró la existencia de un estado de cosas inconstitucional (en adelante ECl); además de ello, expandió sus efectos no solo hacía las decenas de demandantes que habían usado el mecanismo jurisdiccional de tutela individual para remediar la violación a sus derechos fundamentales, sino que dicha macro sentencia alcanzaría a varios millones de personas víctimas del desplazamiento forzado en Colombia.

Dicha sentencia fue la T-025 del año 2004, que no hizo otra cosa que reafirmar lo que en la práctica judicial de diversas cortes se encuentra en ascenso, sobre todo en países con grandes desigualdades sociales, y que en doctrina surge como parte del activismo judicial, me refiero a las denominadas sentencias estructurales.

El Perú no ha sido ajeno a dicha tendencia, pues desde hace más de quince años que viene utilizando la técnica del $\mathrm{ECl}$ en numerosas causas, sobre todo en el último quinquenio, a través del mecanismo de las sentencias estructurales. Si bien a la fecha no se ha expedido alguna de similar envergadura que la T-025; sin embargo, ello no obsta para dejar de afirmar que en nuestro país se viene desarrollando un incipiente modelo jurisdiccional que abarca decisiones de tal naturaleza.

Ahora bien, el objeto del presente trabajo consistirá en describir y analizar diferentes sentencias emitidas por el Tribunal Constitucional peruano que podrían ser calificadas de estructurales, centrándonos en el ámbito de los derechos socioeconómicos.

En tal sentido, la estructura del presente trabajo consistirá en describir, en un primer apartado, algunos conceptos necesarios sobre estado de cosas inconstitucional y la figura de las sentencias estructurales; establecer si ambas figuras tienen características comunes y determinar cuáles son las características propias de las sentencias estructurales. En un segundo apartado, se detallará cuáles son las sentencias estructurales emitidas por el Tribunal Constitucional peruano respecto de los derechos a la salud y a la educación, esto es, en el ámbito de los derechos sociales, para ello se determinará si en el estudio de cada caso se cumplen o no con las características y elementos que configuran una sentencia estructural de acuerdo con lo desarrollado por la doctrina nacional e internacional, así como en la jurisprudencia más relevante de países como los Estados Unidos, Colombia, Sudáfrica y la India.

En la evaluación de cada uno de los apartados del presente trabajo se realizará una reflexión crítica sobre los casos que se comentan, con la única finalidad de tratar de aportar en el desarrollo de esta tendencia en nuestro país. El método empleado es el estudio de casos, así como el uso del método deductivo.

\section{Las sentencias estructurales y sus características}

\subsection{Algunos conceptos necesarios sobre estado de cosas inconstitucional y las sentencias estructurales}

Hace algunas décadas ha empezado a desarrollarse lo que en doctrina se denomina sentencias estructurales o structural remedies, a partir de determinada práctica judicial que ha tenido su origen, según dicha doctrina, en los Estados Unidos de Norteamérica con la Corte de Earl Warren. Pese a que el caso que le dio origen incidió, sobre todo, respecto a derechos civiles, a diferencia del uso que hoy en día se le da respecto a derechos económicos, sociales y culturales, resulta importante destacarlo, pues en gran medida constituye el soporte de lo que actualmente tenemos.

El fallo no viene a ser otro que el caso Brown vs. Board of Education, más específicamente, el conocido como Brown II, de mediados del siglo pasado. Esta sentencia marcó un hito en la historia de los tribunales de justicia, tanto respecto de los derechos fundamentales, como en cuanto a la acción de los tribunales de justicia, pues se apartó de la doctrina "iguales, pero separados"(1), al considerar que la segregación racial en las escuelas era contraria al sentido de la Constitución de los Estados Unidos. Pese a que la demanda fue interpuesta a favor de una sola persona, Linda Brown, el fallo alcanzó a otros niños que se encontraban en similar situación sobre su derecho a la igual protección debido a causas de segregación racial en materia educativa.

El debate que surgió luego del fallo, con relación a los efectos del mismo, dividió 
las posturas, pues, por un lado, neorrealistas como Gerald Rosenberg, negaban que el fallo haya producido los efectos que se tuvo diez años después, a través de políticas y leyes interraciales que implementó el gobierno de los Estados Unidos. De otro lado, la postura constructivista sostenía que el fallo creó un entorno propicio que desencadenó las reformas sociales a favor de la igualdad y contra la segregación racial (Gutiérrez, 2018, p. 289-366).

Esta no sería la única sentencia en la que el máximo tribunal de justicia estadounidense asume una práctica activista, pues según Clara Inés Vargas, citando a Daniel Farber, menciona otros casos que lo siguieron, tales como Swann vs. Charlotte Mecklenburg Board of Education y Pitts vs. Cherry (2003, p. 210).

Dichas sentencias que han sido calificadas de estructurales y que formarían parte del denominado activismo judicial, también han merecido críticas desde quienes consideran que es muy peligroso otorgar mayores competencias a los tribunales de justicia y que carecen de la legitimidad suficiente para emprender tales actuaciones (Bickel, 1962).

Sin ahondar en el debate doctrinario, surgido luego de la sentencia Brown, lo cierto es que los tribunales de justicia son hoy en día más activistas que hace medio siglo, sobre todo, luego de la Segunda Guerra Mundial, periodo en el que el constitucionalismo se ha posicionado como una guía al interior de las constituciones y, por ende, de las actuaciones del Estado y la sociedad. De ahí que un sector de la doctrina afirme que los tribunales tienen la capacidad de transformaciones sociales (Rodríguez Garavito y Rodríguez Franco, 2010, p. 22).

La doctrina también suele destacar las sentencias que a partir de 1997 emite la Corte Constitucional de Colombia, así como la creación de la figura denominada "estado de cosas inconstitucional", figura que ayudó en la construcción de otra denominada "sentencias estructurales".

Así, en la Sentencia de Unificación 559, del 6 de noviembre de 1997, la Corte utilizó, por primera vez, la denominación de estado de cosas inconstitucional (ECI), pero es con la sentencia T-025 del 2004 que logra su máximo desarrollo(2) en la etapa de fortalecimiento (Barriga, 2014, p. 102)(3). De ahí que, en cuanto al concepto de estado de cosas inconstitucional, conforme lo señalará la Corte Constitucional de Colombia en la citada sentencia(4), ha tenido una evolución a nivel jurisprudencial desde la fecha en que se creó dicha figura. Parte de este desarrollo consiste en que las sentencias más recientes consideran que nos encontramos ante un estado de cosas inconstitucional cuando: (i) se presenta una repetida violación de derechos fundamentales de un gran número de personas y (ii) cuando la causa de esa vulneración no es imputable únicamente a la autoridad demandada, sino que reposa en factores estructurales.

En sentido similar, se ha señalado que el $\mathrm{ECl}$ es una figura de carácter procesal, cuyo fin no es únicamente la defensa subjetiva, sino $y$, sobre todo, la defensa objetiva de derechos fundamentales. En dicha figura, el juez declara que existe una situación que lesiona, de manera sistémica y permanente, el ejercicio de los derechos de un número amplio de personas que, en su mayoría, no instauraron la demanda, y en el que advierte problemas estructurales, por lo cual, es necesario involucrar a otros poderes públicos para adoptar las medidas que tiendan a superar ese estado injusto (Vargas, 2003, p. 214).

Ciertamente, el ECl es una figura procesal creada jurisprudencialmente; no obstante, considero que más que un mecanismo, herramienta o técnica usada por los tribunales a fin de expandir los efectos de la sentencia a un número indeterminado de personas, es una situación, un estado que puede o no dar origen a una sentencia estructural, y a través de la cual, un determinado tribunal ${ }^{(5)}$ constata la existencia de problemas que afectan a un mayor número de personas que únicamente a las partes en el proceso, por ello creo que la definición que nos da Alzate resulta más

(1) Fue una doctrina jurídica que recayó en el caso Plessy vs. Ferguson en el que la Corte Suprema de los Estados Unidos, en 1896, decidió en favor de permitir y justificar la segregación racial.

(2) Caso sobre desplazamiento forzado que involucró la afectación de diversos derechos sociales de más de tres millones de colombianos.

(3) La autora considera que han existido, al menos, tres etapas en el desarrollo jurisprudencial de las sentencias estructurales en Colombia, a las que se suele vincular con el uso del ECl:

a) Primera etapa: Surgimiento (1997-1998)

b) Segunda etapa: Consolidación (1998-1999)

c) Tercera etapa: Fortalecimiento (2004-2008)

(4) Tercer párrafo del fundamento 7 de la sentencia T-025 del 22 de enero de 2004.

(5) Existe un consenso en la doctrina de que necesariamente debe ser un alto tribunal de justicia, sea Corte Suprema o un tribunal especializado como el Tribunal Constitucional o la Corte Constitucional. 
precisa. Así, señala que el $\mathrm{ECl}$ es un conjunto de hechos, acciones $\mathrm{u}$ omisiones a través de los cuales se constata una violación masiva de derechos fundamentales. Dichos hechos pueden tener su origen en una autoridad pública específica o en un conjunto de hechos de diversas autoridades que dan origen a un problema sistémico (2004).

Destaco dicho aporte, pues suele vincularse el ECl con las sentencias estructurales, ya que ésta última, más bien calificaría como un tipo de sentencia en la que se usa, de manera excepcional, la figura del ECI. Incluso, la doctrina no las diferencia, pues las considera sujetas a un único mecanismo. Me parece necesaria esta distinción, pues creo que en el ámbito procesal en el que nos encontramos, necesitamos usar conceptos más claros y precisos que nos ayuden a forjar instrumentos que, eventualmente, aportarían a una real protección de los derechos fundamentales. Ello en la medida en que se suele usar dichas figuras de manera imprecisa, e incluso podríamos señalar que existen diversos niveles que podrían o no calificar de sentencias estructurales, pero en los que se ha incluido en casi todos los casos la figura del ECl.

Por ello, cabe la siguiente interrogante: ¿para expedir una sentencia estructural es necesario constatar un estado de cosas inconstitucional? En principio, las sentencias estructurales califican como una modalidad de sentencias. Las sentencias judiciales, en cuanto a sus efectos, pueden ser divididas en aquellas que resuelven y cuyos efectos inciden únicamente en las partes que dieron origen al proceso, mientras existen otras que, por su envergadura, recaen en un número extenso e indeterminado de personas que no participaron del mismo (pudiendo ser decenas, cientos, miles e incluso millones), pero que les afecta de la misma manera que a las partes originarias.

Para la expedición de la sentencia estructural, el tribunal requiere, previamente, constatar una situación generalizada y masiva de violación de derechos (un ECl sin que su origen sea estructural) o, una situación generada por problemas estructurales de derechos fundamentales (un $\mathrm{ECl}$ cuyo origen involucra a más de una entidad estatal y acarrea problemas estructurales de mayor complejidad). En otras palabras, para emitir una sentencia estructural, previamente se requerirá la constatación de un $\mathrm{ECl}$ que genere problemas estructurales o no, pero sí, siempre violación masiva y generalizada de derechos fundamentales. Este estado de cosas será una condición previa y durante el análisis del caso a la decisión que se pretenda tomar, esto es, el ECl causará la emisión de la sentencia estructural.

Con relación estrictamente al $\mathrm{ECl}$, existen sentencias en las que se ha declarado un $\mathrm{ECl}$; sin embargo, no califican de sentencias estructurales, aunque sus efectos se hayan extendido a otras personas no participantes del proceso. La diferencia radica en las causas. En efecto, una sentencia que constata y luego declara un ECl, por ejemplo, no necesariamente puede haberse originado en causas estructurales (que implica, entre otros, la omisión o deficiente desempeño de diversas autoridades públicas para diseñar e implementar políticas públicas), pues la causa podría deberse a que una sola autoridad pública se niegue a hacer efectivo un determinado derecho fundamental a un número indeterminado de personas o que interprete una disposición legal de manera contraria a la Constitución. Con lo cual, la salida para esta última situación sería que dicha autoridad pueda encaminarse en la interpretación que haga viable la protección efectiva del derecho u derechos vulnerados o corregir su actuación u omisión, esto es, la salida es más simple, pues no implica la sujeción de diversas autoridades, lo que haría más dificultoso la ejecución de la sentencia.

Un claro ejemplo recae en la sentencia expedida en el Expediente 02579-2003PHD, caso Arellano Serquén, sobre acceso a la información pública. Aquí lo único que correspondía era que el Consejo Nacional de la Magistratura entregue la información que solicitaban los jueces y fiscales, esto es, no existía ningún problema estructural que involucre a más de una entidad pública o a más de un derecho. Por ello se declaró la existencia de un $\mathrm{ECl}$, pero, a mi juicio, no califica de sentencia estructural.

Así también lo ha entendido la Corte Constitucional de Colombia, al afirmar en la Sentencia T-025 que, regularmente, ha emitido dos tipos de órdenes, dependiendo de la magnitud del problema que genera la vulneración de los derechos que son objeto de tutela. Las que incluyen órdenes de ejecución simple, generalmente referidas a órdenes de abstención o de acción que pueden ser efectuadas por una autoridad sin el concurso de otras, pero también ha dictado órdenes complejas cuyo proceso de ejecución involucran a diversas autoridades y requieren acciones coordinadas (fundamento 10 de la sentencia T-025). Estas últimas son propiamente las que vemos en las sentencias estructurales.

Por ello, me permito coincidir con la posición que asume que las sentencias estructurales son aquellas decisiones 
judiciales que procuran remediar violaciones generalizadas y sistemáticas de los derechos de los ciudadanos, ordenando a las autoridades el diseño y la implementación de políticas públicas (Gutiérrez, 2018, p. 28).

Siguiendo el mismo razonamiento de lo que se ha venido manifestando en el presente trabajo, se ha señalado también que dicho instrumento es un fenómeno polifacético que ha tomado diferentes formas en los sistemas jurídicos en los que ha hecho su aparición. Ninguno de ellos ofrece una muestra suficientemente amplia y detallada que logre capturar la variedad de matices a través de los cuales se ha materializado (Gutiérrez, 2018, p. 31). Lo interesante es que es un instrumento que se encuentra en constante desarrollo y corresponde a la doctrina analizar con mayor detalle su inclusión en la jurisprudencia de diversos países, incluyendo el nuestro.

\subsection{Características comunes de las sentencias estructurales}

Ahora bien, hemos afirmado que existen diferencias entre un $\mathrm{ECl}$ y una sentencia estructural o mejor, que una antecede a la otra, en una estrecha relación de causa-efecto, pero también es cierto que tienen algunas características comunes. De ahí que, cuando la Corte Constitucional de Colombia hace referencia a las características del $\mathrm{ECl}$, no las hace también respecto de las sentencias estructurales, pues en alguna medida considera que abarcan lo mismo.

En la sentencia T-025 del 2004, la referida Corte definió que estamos frente a un estado de cosas inconstitucional cuando convergen: "(i) la vulneración masiva y generalizada de varios derechos constitucionales que afecta a un número significativo de personas; (ii) la prolongada omisión de las autoridades en el cumplimiento de sus obligaciones para garantizar los derechos; (iii) la adopción de prácticas inconstitucionales, como la incorporación de la acción de tutela como parte del procedimiento para garantizar el derecho conculcado; (iv) la no expedición de medidas legislativas, administrativas o presupuestales necesarias para evitar la vulneración de los derechos; (v) la existencia de un problema social cuya solución compromete la intervención de varias entidades, requiere la adopción de un conjunto complejo y coordinado de acciones y exige un nivel de recursos que demanda un esfuerzo presupuestal adicional importante; (vi) si todas las personas afectadas por el mismo problema acudieran a la acción de tutela para obtener la protección de sus derechos, se produciría una mayor congestión judicial”(6).
De forma similar, las sentencias estructurales o "macrosentencias", a decir de Rodríguez Garavito, se caracterizan básicamente por: (i) el tamaño de la población beneficiaria, (ii) la gravedad de las violaciones de derechos que pretende resolver, (iii) los numerosos actores estatales y sociales que involucra, y (iv) la ambición y duración del proceso de implementación de las órdenes del fallo (Rodríguez Garavito y Rodríguez Franco, 2010, p. 14).

Por su parte, Andrés Gutiérrez, ha enfatizado aquellas características que diferencian a las sentencias estructurales de los procesos individuales (2018, p. 39-58), siendo las siguientes:

a) El gran número de personas cuyos derechos son objeto de protección.

Debido a que la protección ordinaria o individual será de poca utilidad, pues deja inalteradas las causas de fondo que provocan la infracción. A su vez, se distingue de la protección de derechos difusos (medioambientales, culturales, etc.), pues, de entrada, estos tienen una vocación netamente global, ya que la titularidad de los derechos que protegen es colectivo y no individual como en el caso de las sentencias estructurales, además, la actuación del juez, definitivamente, afectará a una colectividad. Así, en los casos individuales no existe el denominado giro tutelar ${ }^{(7)}$, en las sentencias estructurales sí.

b) El origen que genera la violación de los derechos: el bloqueo institucional.

La cantidad de afectados demuestra que lo que el tribunal enfrenta no es una causa ordinaria, sino que se trata del denominado bloqueo institucional, esto es, de diversos obstáculos institucionales y culturales que impiden una adecuada reacción del Estado. Se produce una anulación del poder de respuesta del Estado frente a un contexto social crítico. El Estado no está cumpliendo con su rol.

(6) Véase el cuarto párrafo del fundamento 7 de la sentencia T-025 del 2004

(7) Acción de expandir los efectos desde un caso en concreto a otros múltiples y similares al primero o, dicho de otro modo, esto es, la transformación del recurso, marcadamente individualista en su origen, pero que termina desempeñando una función global o estructural a través de la ampliación de los efectos de las decisiones judiciales (Gutiérrez, 2018, p. 56). 
c) La complejidad de las órdenes impartidas.

En esta etapa es importante la creatividad de los jueces para establecer remedios razonables. Así, la complejidad de las sentencias estructurales se traduce en que, en lugar de imponer la ejecución de los mandatos típicos como los de dar, hacer o no hacer, los tribunales de justicia exigen otro tipo de actuaciones que demandarían un mayor esfuerzo institucional de parte de las entidades responsables.

d) Proceso de seguimiento de las sentencias estructurales.

Una vez que se emite la sentencia, concluye el proceso de toma de decisión, pero empieza una fase en la que se evalúa el cumplimiento de las órdenes impartidas. Ello se debe más que nada a la complejidad de las órdenes impartidas y al alto nivel de las entidades a las que se dirigen las sentencias, lo que ha llevado a que, en diversos casos, sean los mismos altos tribunales los que verifiquen el cumplimiento de la sentencia.

Ahora bien, hasta aquí se han detallado las características de las sentencias estructurales, según determinada doctrina y jurisprudencia, en seguida, destaca la siguiente interrogante: ¿Una sentencia debe cumplir estrictamente cada una de las características mencionadas y de manera conjuntiva para ser calificada de estructural?

Siguiendo, nuevamente el trabajo de Andrés Gutiérrez, existen dos visiones distintas que nos dan luz acerca de la citada interrogante (2018, p. 59-63):

a) Toda sentencia que no se restrinja a las partes y que busque ampliar los márgenes de acción para proteger derechos de terceros es una sentencia estructural (el giro tutelar).

b) Para que sea una sentencia estructural, debe cumplir con todos y cada uno de los criterios fijados (todas las características antes desarrolladas).

Coincido en que no necesariamente se deben incluir todos los criterios fijados, pero lo que creo que no debe faltar es el denominado giro tutelar, además de las causas sistémicas, permanentes y estructurales que obedecen normalmente a la actuación u omisión de más de un órgano del Estado en sus diversas instituciones. Por la misma razón, no existe un único modelo que abarque todos los casos. Lo que habría es una idea de gradualidad, dependiendo del caso concreto.
Pero Colombia no es el único país además de los Estados Unidos en utilizar dicha herramienta procesal, otros países que también demuestran altas desigualdades sociales han echado mano de ella. La República de Sudáfrica y la India son dos claros ejemplos. En el primero de ellos, tenemos el caso Grootboom vs. República de Sudáfrica del año 2000. La demanda fue interpuesta por Irene Grootboom y otros, debido a que ella al igual que 900 personas, más de la mitad eran niños, fueron desalojados del terreno que ocupaban de manera precaria, pues durante el periodo del apartheid, muchas familias fueron obligadas a reubicarse en zonas asignadas a ellas, fuera de las ciudades, incluso, abandonando casas y tierras que les habían pertenecido por muchas generaciones.

El desalojo fue llevado a cabo, de forma bastante violenta (quemaron sus casas, e incluso destruyeron sus objetos personales como sus documentos de identificación). En virtud del artículo 26 de la Constitución sudafricana ${ }^{(8)}$, Irene Grootboom planteó una demanda con el fin de acceder a una vivienda adecuada (vivienda digna). La Corte Constitucional de la República de Sudáfrica amparó su derecho y amplió sus efectos a las cerca de 900 personas en la misma situación, pero no dispuso la entrega de viviendas de manera inmediata, sino que ordenó que las autoridades diseñen planes para que las personas afectadas puedan acceder a ellas. La Corte se limitaría a efectuar un control constitucional sobre los planes diseñados ${ }^{(9)}$.

Se advierte desde luego que la sentencia dictada por el tribunal sudafricano recae en una sentencia estructural, pues las causas que dificultaron el acceso a una vivienda digna recayeron básicamente en la omisión del Estado en el diseño e implementación de

(8) El artículo 26 de la Constitución sudafricana establecía con relación al derecho a la vivienda lo siguiente:

a) Todo individuo tiene derecho a acceder a una vivienda adecuada.

b) Estado deberá tomar las medidas legislativas -y de otra índole- razonables en función de los recursos que estuvieran a su alcance para proveer a la concreción progresiva de este derecho.

c) Ningún individuo podrá ser desalojado de su vivienda ni podrá ésta ser demolida sin una orden judicial, la cual deberá ser dictada habiéndose considerado todas las circunstancias relevantes del caso. Ninguna ley permitirá el desalojo arbitrario.

(9) La sentencia completa se encuentra disponible en el portal web de la Corte Constitucional de Sudáfrica (https://collections.concourt. org.za/bitstream/handle/20.500.12144/2107/Full\%20judgment\%20(478\%20Kb)-2798.pdf?sequence=4\&isAllowed=y). Una versión en español se encuentra en la cuenta de academia.edu de María Jimena Sáenz (disponible en file:///C:/Users/sisa2/Downloads/ Caso_Grootboom_Sudafrica.pdf) 
políticas nacionales luego del apartheid y durante el periodo de transición en Sudáfrica, además porque la solución de tal problema correrá a cargo de más de una entidad pública.

De otro lado, tenemos el caso de la India sobre el derecho a la alimentación básica. En el 2001, la Unión del Pueblo para las Libertades Civiles demandó ante el Tribunal Supremo de la India a fin de que diera solución al problema alimenticio de millones de personas de bajos recursos económicos, principalmente debido a las constantes sequías que asolaban al país durante dicho periodo. El Tribunal Constitucional no solo estimó la demanda, sino que fue más allá: identificó el área más perjudicada y ordenó que se proporcione comida a los más vulnerables (niños, mujeres y hombres pobres, ancianos, entre otros), ordenó también que todas las tiendas del sistema público de distribución se volvieran a abrir y se mantengan en funcionamiento, disponiendo, de este modo, órdenes más detalladas con el objeto de salvaguardar el derecho a la alimentación de un amplio sector de la población de la India (Muralidhar, 2013, p. 200-201; Chitalkar y Gauri, 2017, p. 354-357).

Al igual que el caso sudafricano, el tribunal de la India emitió claramente órdenes estructurales. Ambos casos tuvieron como causa común la omisión de las instituciones públicas de implementar políticas nacionales a fin de revertir la situación de violación masiva, permanente y sistémica de derechos sociales de amplios sectores de sus sociedades. Nótese que en ninguno de estos casos se usó la figura del estado de cosas inconstitucional.

\section{Sentencias estructurales del Tribunal Constitucional en el Perú}

Nuestro país no ha sido ajeno a la expedición de sentencias estructurales y a la inclusión de la figura del estado de cosas inconstitucional $(\mathrm{ECl})$. Diversos trabajos e investigaciones han descrito y analizado las sentencias que ha emitido el Tribunal Constitucional, principalmente desde la óptica de la definición de $\mathrm{ECl}$, como Ramírez Huaroto, Dávila-Seminario, Vásquez Armas, Figueroa Gutarra, Hakansson Nieto, entre otros. Otros, en menor medida, han optado por acudir a la figura de las sentencias estructurales, como Barriga Pérez y Rojas Bernal.

Sin perjuicio del análisis que podría efectuarse respecto de si las sentencias en la que se ha declarado un ECl en el Perú, corresponde necesariamente la calificación de estructural, lo cierto es que existen sentencias que han sido consideradas de estructurales pese a que no se incluyó la denominación de estado de cosas inconstitucional. Un claro ejemplo de esto último es la resolución recaída en el Expediente 020022006-PC (caso Pablo Fabián y otros), en la que el Tribunal ordenó que el Ministerio de Salud implemente un sistema de emergencia, dentro de determinado plazo, para atender la salud de las personas contaminadas por plomo en la ciudad de La Oroya, así como el cumplimiento de determinada normatividad que incide en la protección del derecho a la salud debido a problemas medioambientales, entre otras órdenes y exhortaciones hacía otras entidades del Estado.

Ciertamente, tenemos una larga lista de sentencias que en algunos casos podrían ser calificados de estructurales y otros no. Dentro de esta lista, en su mayoría, se incluyen aquellos en los que se ha determinado la vulneración de derechos socioeconómicos de un amplio sector de personas en temas de salud, educación, seguridad social, entre otros.

De otro lado, interesa destacar que no ha habido uniformidad en los criterios para establecer cuándo correspondía emitir una sentencia estructural y cuándo no. Un interesante trabajo sobre ello se encuentra en Rojas Bernal, cuando hace referencia al incipiente activismo dialógico en la emisión de sentencias estructurales en el Perú, estableciendo algunos criterios a los que denomina los puntos críticos de las sentencias estructurales (2017, p. 228-240), los que recaen en la emisión de una sentencia estructural (cuándo acudir a ella y cuándo no), en las órdenes y sus distintas modalidades (órdenes fuertes y débiles) y en la fase de ejecución de sentencias (seguimiento fuerte y seguimiento débil), siguiendo los criterios de Rodríguez Garavito y Rodríguez Franco, en diversas investigaciones.

Ahora bien, nuestro análisis se centrará básicamente en las sentencias estructurales emitidas por el Tribunal Constitucional peruano en el ámbito de los derechos socioeconómicos, particularmente, en dos de ellos: salud y educación, así como determinar cuál ha sido el uso que se ha hecho sobre dicha figura hasta la fecha, a efectos de verificar si su uso continúa en ascenso, si ha habido desarrollo o se encuentra en caída.

\subsection{Sentencias estructurales en materia de salud}

La mayor cantidad de sentencias estructurales que ha dictado nuestro máximo Tribunal Constitucional han recaído en casos sobre el derecho a la salud, tanto en el ámbito de la 
salud física como en el ámbito de la salud mental. Ahora bien, parecería claro que cuando se trata de sentencias estructurales, inmediatamente se reconozca que los derechos económicos, sociales y culturales (DESC) son exigibles judicialmente, en la medida en que para emitir una sentencia estructural es porque previamente se ha verificado la violación masiva de derechos y por tanto, se estime la demanda, esto es, que declare fundada la demanda. Sin embargo, encontramos que existen niveles de su exigibilidad judicial, el cual según Rodríguez Garavito, siguiendo los enfoques judiciales respecto a los DESC desarrollada por Tushnet (2017), podrían clasificarse en "derechos fuertes" y en "derechos débiles".

De otro lado, en el ámbito nacional, el derecho a la salud se encuentra reconocido en distintos dispositivos de la Constitución, cuya protección no solo se limita al ámbito individual, sino que se extiende a la del medio familiar y al de la comunidad (artículo 7), correspondiendo al Estado establecer la política nacional de salud con base en el pluralismo y de forma descentralizada (artículo 9); además, el Estado garantiza el libre acceso (accesibilidad) a prestaciones de salud y supervisa su eficaz funcionamiento (artículo 11). Y pese a que este derecho no está contenido en el capítulo de los derechos fundamentales de la persona (capítulo I), sino en capítulo II, de los Derechos Sociales y Económicos; no obstante, constituye un verdadero derecho fundamental debido a su inescindible conexión con el derecho a la vida (artículo 2), a la integridad (artículo 2) y al principio de dignidad (artículos 1 y 3 ), además porque es una condición indispensable del desarrollo humano y un medio para alcanzar el bienestar del individuo y de la sociedad(10).

En síntesis, el Tribunal Constitucional peruano ha considerado que los derechos económicos y sociales son derechos cuya exigibilidad judicial es aceptable, pero bajo ciertos parámetros.

Las sentencias estructurales en materia del derecho a la salud, conforme se ha señalado antes, han sido emitidas básicamente sobre dos ejes: salud física y salud mental. Con relación a la primera de ellas, tenemos que los primeros dos casos en los que se ha emitido este tipo de sentencias recayeron sobre demandas en las que se solicitaba que el Ministerio de Salud les otorgase a los peticionantes atención médica y un tratamiento integral para combatir el VIH/SIDA, enfermedad que los recurrentes padecían. Las sentencias recayeron en los Expedientes 02945-2003-PA, caso de Azanca Alhelí Meza García ${ }^{(11)}$ y el 02006-2004-PA, caso de José Luis Correa Condori ${ }^{(12)}$.

En ambos casos, muy similares, el Tribunal Constitucional consideró que se justificaba entrar a analizar el fondo de la cuestión, debido no solo a la condición de vulnerabilidad de los demandantes, hecho que justificaba también la razonabilidad de las medidas que al final se tomaron, sino además porque se atentaba contra una pluralidad de derechos relacionales en torno al derecho a la salud, como la vida, integridad y el principio dignidad de la persona. De ahí que, en ambos casos, el efecto expansivo a otros en igual situación que los demandantes (giro tutelar), se haya producido con la exhortación a los poderes públicos para que den cumplimiento del artículo 8 de la Ley 26626.

En efecto, pese a que en este caso el Tribunal peruano no declaró la existencia de un estado de cosas inconstitucional, sin embargo, exhortó a los poderes públicos a que den cumplimiento al artículo 8 de la Ley 26626, modificada por la Ley 28243 , que establece que la protección del derecho a la salud alcanza a la entrega de retrovirales de manera gratuita a personas en situación de vulnerabilidad y pobreza extrema y de manera progresiva, a ello añade el tema presupuestario, ya que considera que debe considerarse el Plan de Atención de Lucha contra el Sida uno de carácter prioritario en materia presupuestal. Por este simple hecho, se infiere que la exhortación no solo está dirigida a la demandada, el Ministerio de Salud, sino además a otras entidades del Estado como el Ministerio de Economía y Finanzas (MEF), a efectos de que la entrega de retrovirales se haga efectiva no solo para el caso de ambos demandantes, sino también para personas que se encuentran en igual condición, esto es, con VIH y en situación de vulnerabilidad o en extrema pobreza.

Otro caso en el que si bien el tema medular recayó sobre un derecho colectivo (medioambiente), pues se verificó que personas de la ciudad de La Oroya se encontraban con altos porcentajes de plomo en sus organismos debido a la contaminación de un complejo metalúrgico a cargo de la empresa DoeRun Perú SRL; finalmente, se definió en torno al derecho a la salud debido, concretamente, a la contaminación y al daño

(10) Tribunal Constitucional del Perú, sentencia STC Expediente 1429-2002-HC.

(11) Publicada en el portal web del Tribunal Constitucional el 12 de julio de 2004

(12) Publicada en el portal web del Tribunal Constitucional el 8 de abril de 2005. 
en la salud y a la integridad de aquellas personas. Se trata de la sentencia recaída en el Expediente 02002-2006-PC, caso Pablo Fabián y otros ${ }^{(13)}$. En este tampoco se reconoció la figura del estado de cosas inconstitucional; sin embargo, los hechos que dieron origen al problema, la evidente vulneración masiva de derechos y la magnitud del problema medioambiental generado, impulsaron a que se tomará una decisión estructural para beneficiar a la población en su conjunto (Barriga, 2017, p. 252).

Cabe destacar, que en este caso, entre otros, se ordenó que el Ministerio de Salud implemente un sistema de emergencia para atender la salud de las personas contaminadas por plomo, otorgando un plazo para ello de treinta días; que la Dirección General de Salud Ambiental (DIGESA) del Ministerio de Salud, en el mismo plazo, realice todas aquellas acciones tendentes a la expedición del diagnóstico de línea base, a fin de implementar los respectivos planes de acción para el mejoramiento de la calidad del aire en la ciudad de La Oroya conforme al Decreto Supremo 074-2001-PCM; que el citado ministerio y en el mismo plazo, realice todas las medidas tendentes a declarar el estado de alerta en la ciudad de La Oroya, conforme al referido decreto supremo y a la Ley 26842. De otro lado, se exhortó a otras entidades del Estado: Gobierno Regional de Junín, Municipalidad Provincial de Yauli-La Oroya, Ministerio de Energía y Minas, Consejo Nacional del Ambiente y a las empresas privadas que desarrollan actividad minera en La Oroya, que participen en las acciones pertinentes que permitan la protección de la salud de los pobladores de La Oroya.

Así, se involucró a distintas entidades estatales, además de emitir órdenes precisas relativas a políticas públicas necesarias para superar la situación de violación del derecho a la salud de las personas que habitan la ciudad de La Oroya.

Por otro lado, tenemos el grupo de casos en los que el Tribunal Constitucional emitió sentencias estructurales en el ámbito del derecho a la salud mental. El primero de ellos, fue uno sobre salud mental de personas privadas de su libertad, en el caso de Pedro Gonzalo Marroquín, recaído en el Expediente 3426-2008-PHC/TC ${ }^{(14)}$. Nuevamente, se incide sobre un derecho social: la salud. Esta sentencia es interesante en la medida en que el giro tutelar se da después de verificar con datos concretos que, efectivamente, existían otras personas en idéntica situación que el demandante (persona que padecía de esquizofrenia y que encontrándose privada de su libertad mediante condena, tenía una orden judicial para que sea internado en algún centro hospitalario). En efecto, se había verificado que existían alrededor de 71 personas en la misma situación. En el caso concreto del demandante, los hospitales a los que acudía la demandada, el Instituto Nacional Penitenciario, denegaban su acceso debido a cuestiones técnicas, como la falta de camas, por ejemplo.

En este caso, además de declarar la existencia de un estado de cosas inconstitucional, la falta de una política de tratamiento y rehabilitación de la salud mental de personas que se encuentran sujetas a medidas de seguridad de internación por padecer de una enfermedad mental, el máximo tribunal de justicia en materia constitucional ordenó al Ministerio de Economía y Finanzas para que adopte las medidas necesarias que permitan el incremento gradual del presupuesto destinado al Ministerio de Salud, concretamente, a los centros hospitalarios de salud mental del país; ordenó al Poder Judicial la adopción de las medidas correctivas para que todos los jueces del país emitan pronunciamiento oportuno sobre los informes médicos que les son remitidos por las autoridades de salud, que recomiendan el cese de la medida de seguridad de internación; exhortó al Congreso de la República para que proceda a la aprobación de una ley que regule el tratamiento, supervisión, procedimiento, ejecución y cese de las medidas de seguridad de internación; y, exhortó al Poder Ejecutivo para que adopte las medidas necesarias que tengan por objeto superar de manera inmediata y eficaz las situaciones de hecho que dan lugar al quebrantamiento de la Constitución, fortaleciendo los niveles de coordinación con el Ministerio de Justicia, Ministerio de Salud, Ministerio de Economía y Finanzas, entre otros.

El último caso en el que el Tribunal Constitucional declaró la existencia de un estado de cosas inconstitucional y emitió una sentencia estructural por un tema de salud mental, recayó en el Expediente 04007-2015-PA, caso de la persona de iniciales M.H.F.C. El objeto de la demanda de hábeas corpus dirigida contra el director del Instituto Nacional Penitenciario (INPE) era que se disponga su traslado desde el establecimiento penitenciario de Lurigancho a un centro psiquiátrico especializado, puesto que luego de ser sentenciado a diez años de

(13) Publicada en el portal web del Tribunal Constitucional el 27 de junio de 2006 y en el diario oficial El Peruano el 5 de agosto de 2006.

(14) Publicado en el portal web el 2 de setiembre de 2010 y en el diario oficial El Peruano el 3 de noviembre de 2010. 
Las sentencias estructurales del Tribunal Constitucional peruano en el ámbito de los derechos socioeconómicos: salud y educación

The structural judgments of the Peruvian Constitutional Court in the field of socioeconomic rights: health and education

pena privativa de libertad por homicidio y ya recluido, empezó a padecer de esquizofrenia paranoide.

En la sentencia se consideró que la situación del demandante era representativa de todo un grupo de personas que, estando internadas en alguno de los establecimientos penitenciarios del país, tienen problemas de salud mental, con lo cual, se optó no solo por declarar fundada la demanda, sino, además, por emitir una sentencia estructural luego de declarar un estado de cosas inconstitucional.

En este caso, el giro tutelar se habría dado debido a las siguientes causas: (i) Según informe del INPE de las 114,583 personas recluidas en establecimientos penitenciarios, 457 internos registraban problemas de salud mental; (ii) a fin de tratar a dicho grupo en situación de vulnerabilidad, se cuenta con un solo psiquiatra a nivel nacional, el que presta servicio de manera itinerante; (iii) el INPE no cuenta con un protocolo para la detección y tratamiento del interno con discapacidad mental; (iv) los psicólogos, en su mayoría, atienden programas de resocialización con inclinación educacional, (v) no existe el servicio de psicología clínica; (vi) existen diversas carencias materiales, estructurales y normativas a nivel del INPE y el MINSA, (vii) el servicio de psiquiatría del EP Lurigancho es el único penal, a nivel nacional, que cuenta con camas para hospitalización para este tipo de pacientes, (viii). el Ministerio de Salud no acepta para su hospitalización a los pacientes con trastornos mentales provenientes del INPE, aún existiendo una orden judicial (Sánchez, 2019, p. 115).

Las órdenes emitidas involucraron a distintas entidades del Estado. Así, se ordenó al Ministerio de Justicia y Derechos Humanos y al INPE, en coordinación con el Ministerio de Salud, diseñar, proponer y ejecutar un plan de acción que, en el plazo máximo que vencerá el 6 de enero de 2021, pueda asegurar la disponibilidad y accesibilidad del servicio de salud mental de las personas privadas de su libertad a nivel nacional; se ordenó al INPE que elabore, en coordinación con el Ministerio de Salud, en un plazo máximo de tres meses, contados desde la fecha de publicación de la sentencia, un protocolo para la detección y tratamiento de los internos que padecen de enfermedades mentales; se ordenó al INPE que, en coordinación con el Ministerio de Salud, en un plazo máximo de doce meses, contados desde la fecha de publicación de la sentencia, identifique el total de la población carcelaria que padece de algún tipo de trastorno mental; $y$, se dispuso que el Poder Ejecutivo, en coordinación con el Poder Legislativo, puedan realizar las gestiones pertinentes que tiendan al aseguramiento presupuestario de dicho plan de acción.

A la fecha, no se tiene información acerca si dichas sentencias se habrían cumplido, aunque con relación al primer caso referido a salud mental, siendo uno del 2008, se encuentra en un trabajo previo (2019). Por ello, resulta necesario realizar un estudio que profundice los niveles de cumplimiento de las sentencias estructurales en el ámbito de los derechos socioeconómicos en nuestro país.

\subsection{Sentencias estructurales en materia de educación}

El 17 de junio de 2010, el Tribunal Constitucional emitió la que sería la primera sentencia estructural sobre el derecho a la educación en el Perú. Resulta interesante en la medida en que esta se dictó al interior de un proceso de inconstitucionalidad a diferencia de su par, la Corte Constitucional colombiana, cuyas sentencias estructurales se dictan sobre todo en procesos de tutela. La demanda de inconstitucionalidad fue interpuesta por más de 5000 ciudadanos contra la Ley 28564, norma que derogó la Ley 27504 y restituyó el tercer párrafo del artículo 5 de la Ley Universitaria.

En este caso, la sentencia, además de estimar la demanda, consideró que existía un estado de cosas inconstitucional en el sistema universitario peruano debido a que se acreditó la presencia de elementos objetivos que permitieron concluir la existencia de una profunda crisis, pues el Estado omitió cumplir con su deber constitucional de garantizar una educación universitaria de calidad ${ }^{(15)}$.

En este proceso de inconstitucionalidad, no se produjo el denominado giro tutelar, pues desde el comienzo se destacó la situación crítica de todo el sistema educativo universitario en el país; además, en dicho tipo de proceso sobre inconstitucionalidad, lo que hace el Tribunal es un control abstracto de disposiciones, cuyo pronunciamiento, definitivamente, incidirá sobre una gran cantidad, definida o indefinida, de personas, en este caso, sobre quienes son estudiantes universitarios o sobre quienes pretenden acceder al sistema educativo superior universitario.

Para ello, el alto tribunal destacó algunos datos interesantes ${ }^{(16)}$, entre ellos, que el Perú

(15) Fundamento 210 de la sentencia recaída en el Expediente 00017-2008-PI.

(16) Fundamento 214 de la sentencia recaída en el Expediente 00017-2008-PI. 
ocupa uno de los niveles más bajos del mundo en cuanto a calidad educativa, se encuentra en el puesto 133 de 134 países evaluados, el presupuesto destinado a la educación ha ido decreciendo año tras año, entre otros. Todo ello permitió corroborar la existencia de un estado de cosas inconstitucional de carácter estructural en el sistema educativo universitario(17).

Ahora bien, el Tribunal Constitucional no solo consideró la existencia de problemas de carácter estructural, sino que además dispuso una serie de órdenes precisas, entre ellas, dispuso la creación de una Superintendencia altamente especializada, objetivamente imparcial y fiscalizada por el Estado, lo que hoy en día conocemos como la Superintendencia Nacional de Educación Superior Universitaria (SUNEDU), creada a raíz de la sentencia que aquí se comenta. Es más, precisó cuáles deberían ser las funciones de tal órgano en el sistema educativo superior: (i) evaluar a todas las universidades del país, incluyendo a sus filiales; (ii) evaluar a todas las universidades y sus filiales ratificadas o autorizadas por el Consejo Nacional de Autorización y Funcionamiento de las Universidades (CONAFU); y (iii) garantizar que el examen de admisión a las universidades cumpla con los niveles adecuados de exigibilidad y rigurosidad intelectual.

Resulta loable que el Tribunal Constitucional haya dictado sendas sentencias estructurales sobre el derecho social a la educación en sus dos etapas: el caso antes descrito con relación a la educación superior universitaria y la que a continuación se analizará con relación con la educación básica: inicial, primaria y secundaria.

El caso que se comentará ha sido calificado por la actual presidenta del Tribunal Constitucional como un caso dramático y conmovedor de un país que se encuentra muy cerca de celebrar los doscientos años de la instauración de su República (Ledesma y Sánchez, 2019, p. 623). Es el caso de las hermanas Cieza Fernández, sentencia recaída en el Expediente 00853-2015-PA(18). Se trata de dos hermanas, Marleni y Elita Cieza Fernández, de dieciocho y diecinueve años, respectivamente, que pretendían acceder al primer grado de educación secundaria en un centro de educación básico regular (CEBR), y pese a la anuencia del director de la escuela, autoridades de la Unidad de Gestión Educativa del Ministerio de Educación no les permitían dicho acceso, ya que por sus edades, correspondía ser matriculadas en un centro de educación básica alternativa (CEBA); sin embargo, para que pueden asistir a este último tenían que desplazarse desde el caserío en el que vivían (La Flor, distrito La Cumba, provincia de Utcubamba, región Amazonas) durante aproximadamente cuatro horas: dos horas a pie y dos horas en bus hasta Bagua Grande, lugar más cercano en el que se había instalado un centro de educación básica alternativa con horario nocturno.

El Tribunal Constitucional constató la existencia de un estado de cosas inconstitucional la situación de las hermanas Cieza Fernández como una situación representativa de miles de personas que residen en el ámbito rural y en estado de extrema pobreza(19). Para ello se valió de fuente estadística del Instituto Nacional de Informática y Estadísticas (INEI), de donde obtuvo que hacía el año 2015 existían cinco grupos de departamentos con niveles de pobreza extrema, en el primer grupo destacó la región Cajamarca con una tasa de pobreza extrema entre $16.6 \%$ a $23.9 \%$, mientras que en el segundo grupo se encontraba la región Amazonas, lugar en el que vivían las demandantes, además de Ayacucho y Huancavelica con una tasa de pobreza extrema entre el $8.8 \%$ al $12.3 \%{ }^{(20)}$.

Del mismo modo que en el caso anterior, el fallo determinó órdenes precisas y con plazos específicos de cara al bicentenario de nuestro país. Así, ordenó que el Ministerio de Educación diseñe y ejecute un plan de acción en el plazo de cuatro años, que vencería el 28 de julio de 2021, para garantizar la disponibilidad y accesibilidad a la educación de niños, adolescentes y mayores de edad de extrema pobreza del ámbito rural, empezando por los departamentos de Cajamarca, Amazonas, Ayacucho y Huancavelica. Dispuso que el Poder Ejecutivo en coordinación con el Poder Legislativo realice las gestiones pertinentes que tiendan al aseguramiento de dicho plan de acción.

Existen otros ámbitos en los que el Tribunal Constitucional habría emitido sentencias estructurales: seguridad social, identidad cultural, entre otros, sobre todo en el último

(17) Fundamento 217 de la sentencia recaída en el Expediente 00017-2008-PI

(18) Publicada en el portal web del Tribunal Constitucional el 18 de setiembre de 2017 y en el diario oficial El Peruano el 7 de noviembre de 2017.

(19) Fundamento 61 de la sentencia recaída en el Expediente 00853-2015-PA

(20) Fundamento 74 de la sentencia recaída en el Expediente 00853-2015-PA. 
quinquenio, con lo cual, resulta plausible afirmar que el uso de dicha herramienta procesal va en ascenso en nuestro país. Por lo que queda pendiente analizar todas sus formas y usos, así como la efectiva implementación de dichas sentencias.

\section{Conclusiones}

Estando así las cosas resulta importante establecer enunciativamente algunas conclusiones a las que se ha arribado en el presente trabajo:

- Es posible afirmar que el activismo judicial de los tribunales de justicia forma parte de lo que hoy en día denominamos constitucionalismo y que ha ido desarrollándose en diferentes países, sobre todo después de la Segunda Guerra Mundial. Uno de los elementos de ese activismo es la creación de figuras de orden procesal y sustancial como las sentencias estructurales.

- Dicha herramienta surge a partir de la necesidad de incluir en una sola sentencia la situación de decenas, cientos, miles o millones de personas que ven violados determinados derechos fundamentales a causa de la inoperancia del Estado, esto es, debido a problemas permanentes, sistémicos y estructurales y que han tenido como punto de partida una demanda de naturaleza individual.

- Así también, a través de ella se consolida la facultad contralora y revisora de la conformidad de las políticas públicas que, objetivamente, en nuestro país, al no ser correctamente diseñadas e implementadas luego, generan impunemente violación masiva y generalizada de derechos fundamentales.

- Los países que han utilizado dicha técnica, sin importar la denominación que les dan, estructural remedies, sentencias estructurales, estado de cosas inconstitucional, entre otras denominaciones, se han caracterizado por ser países con grandes desigualdades sociales: Estados Unidos con el racismo, Colombia, Sudáfrica y la India con desigualdades socioeconómicas. El Perú también es un país con grandes desigualdades sociales, de ahí que desde hace más de quince años que las diferentes conformaciones del Tribunal Constitucional han venido usando dicha figura, aunque con mucho temor y desconfianza.

- Debido a las diferencias normativas, sociales y culturales de cada país que la ha venido aplicando, no existe un estándar respecto de las características y elementos que conllevan las sentencias estructurales, ha sido la doctrina, sobre todo norteamericana, colombiana, africana y de la India, las que han analizado la situación de tal herramienta.

- En nuestro país, su uso ha recaído sobre todo sobre derechos socioeconómicos: salud, educación, seguridad social, identidad cultural, etc., pero pese a que han transcurrido más de quince años desde que se empezó a utilizar dicha figura, aún nos encontramos en un estado incipiente respecto de su desarrollo. No sabemos si ha habido cumplimiento parcial o total de las sentencias dictadas a la fecha, por ende, no sabemos si dichas sentencias han tenido resultados positivos a fin de lograr el tan ansiado ejercicio efectivo de los derechos de decenas, cientos, miles o millones de personas.

- Dos de los derechos en los que en mayor medida se han dictado dicho tipo de sentencias han sido salud y educación. Con relación al primero de ellos, se han dictado distintas sentencias en el ámbito de la salud física y la salud mental, en este último caso, de personas privadas de su libertad o con alguna medida de seguridad. Con relación al derecho a la educación, el Tribunal Constitucional ha dictado sentencias estructurales destacando dos etapas del proceso educativo, uno que abarca la educación básica (primaria y secundaria) con el caso de las hermanas Cieza Fernández, y el segundo recayó en la educación universitaria en el que se declaró la existencia de un estado de cosas inconstitucional debido a la crisis del sistema educativo por la baja calidad académica de las universidades del país.

\section{Referencias bibliográficas}

Abramovich, Víctor y Courtis, Christian (2002). Los derechos sociales como derechos exigibles. Madrid: Editorial Trotta.

Alzate, Luis (2004). El estado de cosas inconstitucional. Revista Internauta de Práctica Jurídica, (13). Recuperado el 8 de febrero de 2020, de http://www.egov.ufsc.br/portal/conteudo/ el-estado-de-cosas-inconstitucional.

Barriga, Mónica (2017). Estados de cosas inconstitucionales. Análisis y balance de la jurisprudencia del Tribunal Constitucional. Cuadernos sobre jurisprudencia constitucional, 12, p. 241-256.

(2014). Sentencias estructurales y protección del derecho a la salud. (Tesis de postgrado). Pontificia Universidad Católica del Perú. Recuperado de http://tesis.pucp.edu.pe/ repositorio/handle/20.500.12404/5438 
Chitalkat, Poorvi y Gauri, Varun (2017). India: cumplimiento de las órdenes sobre el derecho a la alimentación. En M. Langford, y R. Garavito y J. Rossi (edit.) La Lucha por los Derechos Sociales. Los fallos judiciales y la disputa política por su cumplimiento (p. 344-374). Bogotá: Editorial De Justicia.

Dávila-Seminario, Carmen (2018). Legitimidad del Tribunal Constitucional para declarar un estado de cosas inconstitucional: alcances y límites de dicha atribución. (Tesis para optar el título de abogada). Lima. Recuperado de https://pirhua.udep.edu.pe/ handle/11042/3653

Gargarella, Roberto (2014). EI nuevo constitucionalismo dialógico frente al sistema de frenos y contrapesos. Por una justicia dialógica. El Poder Judicial como promotor de la deliberación democrática. Buenos Aires: Editorial siglo XXI.

Gutiérrez, Andrés (2018). El amparo estructural de los Derechos. Centro de Estudios Constitucionales (CEPC), Madrid.

Langford, Malcolm y Kahanovitz, Steve (2017). Sudáfrica: repensar las narrativas sobre el cumplimiento. En M. Langford, C. Rodríguez y J. Rossi (edit.), La Lucha por los Derechos Sociales. Los fallos judiciales y la disputa política por su cumplimiento (p. 376-416). Bogotá: Editorial De Justicia.

Ledesma, Marianella y Sánchez, Isabel (2019). El caso de las hermanas Cieza (STC 00853-2015-PA/ TC) desde la perspectiva del derecho fundamental a la educación: un antes y un después en la jurisprudencia del Tribunal Constitucional. En M. Ledesma (coord.), Justicia y Educación. Saberes y prácticas inclusivas en América y Europa (p. 623-641). Lima: Centro de Estudios Constitucionales.

Muralidhar, S. (2013). India: Las expectativas y los retos del cumplimiento judicial de los Derechos Sociales. En M. Langford (edit.), Teoría y Jurisprudencia de los Derechos Sociales: Tendencias emergentes en el Derecho Internacional y Comparado (p. 181-210). Bogotá: Siglo del Hombre editores.

Ramírez, Beatriz (2013). El estado de cosas inconstitucional y sus posibilidades como herramienta para el litigio estratégico de derecho público. Una mirada a la jurisprudencia peruana y colombiana. (Tesis de postgrado). Pontificia Universidad Católica del Perú. Recuperado de http://tesis.pucp.edu.pe/repositorio/handle/20.500.12404/4952

Rodríguez, César y Rodríguez, Diana (2010). Cortes y Cambio Social. Cómo la Corte Constitucional transformó el desplazamiento forzado en Colombia. Colección De Justicia. Bogotá: Ediciones Antropos.

Rojas, José Miguel (2017). Nuestro incipiente 'activismo dialógico': las sentencias estructurales del Tribunal Constitucional. Cuadernos sobre jurisprudencia constitucional, 12, p. 221-240.

Sánchez, Isabel (2019). El derecho a la educación de las personas en situación de pobreza extrema en el ámbito rural. El Estado de Cosas Inconstitucional en el caso de las hermanas Ciez. Gaceta Constitucional, Tomo 142, p. 133-140.

Sánchez, Silvia (2019). Salud mental de personas privadas de libertad o con medida de seguridad y la declaración de estado de cosas inconstitucional. Gaceta Constitucional, Tomo 142, p. 111-123.

Vargas, Clara Inés (2003). La garantía de la dimensión objetiva de los derechos fundamentales y la labor del juez constitucional colombiano en sede de acción de tutela: el llamado estado de cosas inconstitucional. Revista del Centro de Estudios Constitucionales, 1(1), p. 203-228. Recuperado de https://www.redalyc.org/pdf/820/82010111.pdf 